\title{
Finite Volume Scheme Based on Cell-vertex Reconstructions for Anisotropic Diffusion Problems with Discontinuous Coefficients
}

\author{
Ricardo Costa $^{1}$ and Stéphane Clain ${ }^{1,2}$ and Gaspar J. Machado ${ }^{1}$ \\ 1 Centre of Mathematics, University of Minho, Campus of Azurém, 4800-058 \\ Guimarães, Portugal \\ pg24046@alunos.uminho.pt, $\{$ clain,gjm $\} @$ math.uminho.pt \\ 2 Institut de Mathématiques de Toulouse \\ Université Paul Sabatier, 31062 Toulouse, France
}

\begin{abstract}
We propose a new second-order finite volume scheme for non-homogeneous and anisotropic diffusion problems based on cell to vertex reconstructions involving minimization of functionals to provide the coefficients of the cell to vertex mapping. The method handles complex situations such as large preconditioning number diffusion matrices and very distorted meshes. Numerical examples are provided to show the effectiveness of the method.
\end{abstract}

Keywords: Finite volume, second-order, non-homogeneous and anisotropic diffusion

\section{Introduction}

The finite volume method for the linear diffusion equation is an important building-block to solve more complex models such as the Navier-Stokes equations and nonlinear coupling problems. Major efforts have been made to design very high-order schemes up to sixth-order (see $[6,10,12,13,20]$ and the references herein). Nevertheless, the design of second-order schemes is still a challenging and important question for several reasons: very high-order methods are rather complicated and require an important implementation effort whereas secondorder methods are quite simple and easy to code.

A popular class of second-order finite volume schemes is based on vertex reconstructions using point-wise approximations on cells associated to a specific point location (usually the centroid). Then combining cell and vertex values, gradient approximations are evaluated to compute the diffusive flux on the interfaces. There exists an important literature on the subject to provide the gradient approximations but there are very few studies about the way to provide the nodal values, in particular, when dealing with the boundary conditions (essential or natural).

To sum-up, there exist two main techniques to determine the values at the vertices from the values associated to the cells: the Frink-Rauch-BatinaYang $[14-17,11,5]$ way based on the minimization of the coefficients of a linear 
combination (one for each cell in the stencil), and the Coudière-Vila-VilledieuBertolazzi-Manzini $[2,3,7,9,18,19]$ way based on the minimization of the coefficients of a first-degree polynomial. From our point of view, the latter one presents a major drawback since the preservation of the positivity principle cannot be guaranteed.

We have considered the Frink-Rauch-Batina-Yang's method where a more general technique to provide the coefficients is introduced as well as the notion of target combination [4], dedicated to homogeneous and isotropic situations, i.e., the coefficients are simple constant values.

The present work intends to extend the previous study to non-homogeneous and anisotropic problems where the diffusion coefficient is given by a nonconstant matrix. Such situations give rise to new specific numerical difficulties when the eigenvalues of the diffusion matrix are very different leading to numerical locking. Another aspect is the maximum principle preservation which is not fulfilled by the most classical schemes $[23,21,1]$. The present scheme succeed in maintaining the positivity of the solution and the maximum principle is achieved in all the tested cases.

The paper is organized as follows. After the introduction of the generic finite volume scheme for the anisotropic diffusion problem in Sect. 2, we address the cell to vertex mapping and the polynomial reconstruction issues in Sect. 3. We devote Sect. 4 to the details on the finite volume discretization. Numerical tests are given in Sect. 5 and the study ends with a conclusion and some perspectives.

\section{Finite Volume for Anisotropic Diffusion Problems}

Let $\Omega$ be an open bounded polygonal domain of $\mathbb{R}^{2}$ with boundary $\partial \Omega$. Since we shall consider situations where the diffusion tensor may comprise discontinuities, we split $\Omega$ into to two non-overlapping subdomains $\Omega_{1}$ and $\Omega_{2}$ such that $\Omega=$ $\Omega_{1} \cup \Omega_{2}$ sharing a common interface $\Gamma$ where the discontinuity is imposed. Notice that we only consider one discontinuity in order to simplify the presentation but one can easily generalize the method to a greater number of discontinuities. We denote by $K_{1} \equiv K_{1}(x, y)$ and $K_{2} \equiv K_{2}(x, y)$ the diffusion (or permeability) tensors in $\Omega_{1}$ and $\Omega_{2}$, respectively, which can be represented by a $2 \times 2$ symmetric and strictly positive definite real matrices. We seek function

$$
\phi \equiv \phi(x, y)=\left\{\begin{array}{l}
\phi_{1} \equiv \phi_{1}(x, y), \text { in } \Omega_{1}, \\
\phi_{2} \equiv \phi_{2}(x, y), \text { in } \Omega_{2},
\end{array}\right.
$$

solution of the anisotropic steady-state diffusion equations

$$
\begin{array}{ll}
\nabla \cdot\left(-K_{1} \nabla \phi_{1}\right)=f_{1}, & \text { in } \Omega_{1}, \\
\nabla \cdot\left(-K_{2} \nabla \phi_{2}\right)=f_{2}, & \text { in } \Omega_{2},
\end{array}
$$

where the source terms $f_{1} \equiv f_{1}(x, y)$ and $f_{2} \equiv f_{2}(x, y)$ are regular functions on $\bar{\Omega}_{1}$ and $\bar{\Omega}_{2}$, respectively. When dealing with a discontinuity, we prescribe the continuity condition,

$$
\phi_{1}=\phi_{2}, \quad \text { on } \Gamma \text {. }
$$


The boundary $\partial \Omega$ is also partitioned into two subsets $\Gamma_{\mathrm{D}}$ and $\Gamma_{\mathrm{N}}$ such that $\partial \Omega=\Gamma_{\mathrm{D}} \cup \Gamma_{\mathrm{N}}$, in order to prescribe the Dirichlet and the Neumann conditions

$$
\phi=\phi_{\mathrm{D}}, \quad \text { on } \Gamma_{\mathrm{D}}, \quad-K \nabla \phi \cdot n=g_{\mathrm{N}}, \quad \text { on } \Gamma_{\mathrm{N}},
$$

respectively, where $\phi_{\mathrm{D}} \equiv \phi_{\mathrm{D}}(x, y)$ and $g_{\mathrm{N}} \equiv g_{\mathrm{N}}(x, y)$ are given regular functions, and $n$ denotes the unit normal to $\partial \Omega$ outward to $\Omega$. The diffusion tensor $K$ stands for $K_{1}$ or $K_{2}$ depending on the domain. We assume that either $\Gamma_{\mathrm{D}}$ or $\Gamma_{\mathrm{N}}$ may be empty.

\subsection{Mesh}

To design the numerical schemes, we denote by $\mathcal{T}$ a mesh of $\Omega$ constituted of $I$ non-overlapping convex polygonal cells $c_{i}, i=1, \ldots, I$, and $N$ vertices $v_{n}$, $n=1, \ldots, N$. We adopt the following conventions (see Fig. 1 ) we detail hereafter:

- for any cell $c_{i}$ we represent by $\partial c_{i}$ its boundary and by $\left|c_{i}\right|$ its area; we denote by $q_{i}, b_{i}$, and $m_{i}$ a generic point, the centroid, and the centre of mass of $c_{i}$, respectively;

- two cells $c_{i}$ and $c_{j}$ share a common edge $e_{i j}$ whose length is $\left|e_{i j}\right|$, the midpoint is $m_{i j}$ and $n_{i j}$ is the unit normal vector to $e_{i j}$ outward to $c_{i}$, i.e. $n_{i j}=-n_{j i}$; if an edge of $c_{i}$ belongs to the boundary $\partial \Omega$, we replace the index $j$ by $\mathrm{D}$ or $\mathrm{N}$ if $e_{i j}$ belongs to $\Gamma_{\mathrm{D}}$ or $\Gamma_{\mathrm{N}}$, respectively;

- for any cell $c_{i}$ we associate the index set $\nu(i) \subset\{1, \cdots, I\} \cup\{\mathrm{D}, \mathrm{N}\}$ such that $j \in \nu(i)$ if $e_{i j}$ is a common edge of $c_{i}$ and $c_{j}$ or with the boundary $\Gamma_{j}$ if $j=\{\mathrm{D}, \mathrm{N}\}$.

Remark 1. If $v_{n}$ is a vertex at the intersection of $\partial \Omega$ and $\Gamma$, we assume that $v_{n}$ belongs to $\partial \Omega$ and will be treated as a Dirichlet or Neumann point depending on the boundary partition. Moreover, if $v_{n}$ is a vertex at the intersection of $\Gamma_{\mathrm{D}}$ and $\Gamma_{\mathrm{N}}$, we consider that $v_{n}$ belongs to $\Gamma_{\mathrm{D}}$ and, therefore, will be treated as a Dirichlet point.

\subsection{Generic Finite Volume Scheme}

To provide the finite volume scheme, equations (1a) and (1b) are integrated over each cell of the mesh and applying the divergence theorem we get

$$
\sum_{j \in \nu(i)} \frac{\left|e_{i j}\right|}{\left|c_{i}\right|} \frac{1}{\left|e_{i j}\right|} \int_{e_{i j}}\left(-K \nabla \phi \cdot n_{i j}\right) \mathrm{d} s-\frac{1}{\left|c_{i}\right|} \int_{c_{i}} f \mathrm{~d} A=0 .
$$

Let $\phi_{i}$ be an approximation of $\phi$ on $q_{i}$ and let us gather all the approximations in vector $\Phi=\left(\phi_{i}\right)_{i=1, \ldots, I}$. We then substitute the exact scheme (3) by a secondorder accuracy numerical scheme, with respect to the mesh parameter $h_{\mathcal{T}}$, and depending on vector $\Phi$,

$$
\sum_{j \in \nu(i)} \frac{\left|e_{i j}\right|}{\left|c_{i}\right|} \mathcal{F}_{i j}(\Phi)-f_{i}=\mathcal{O}\left(h^{2}\right),
$$

where $\mathcal{F}_{i j}$ is an approximation of the diffusive flux through the edge $e_{i j}$ and $f_{i}$ is an approximation of the mean value of $f$ over the cell $c_{i}$. 


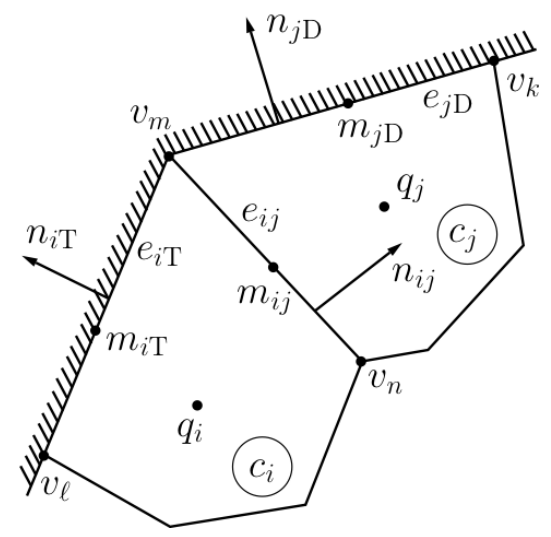

Fig. 1. Mesh notations.

\section{Cell-vertex Mapping and Interpolation}

We want to design a second-order finite volume scheme using both approximations on the cells and on the vertices where the unknowns are only located on the cells. It results that an accurate evaluation of the vertex values with respect to the cell values must be implemented. Let $\psi_{n}, n=1, \ldots, N$, be an approximation of $\phi$ on vertex $v_{n}$ and let us gather all these approximations in vector $\Psi=\left(\psi_{n}\right)_{n=1, \ldots, N}$. The goal of this section is the design of a procedure to compute $\Psi$ from $\Phi$.

\subsection{The Stencils and the Data}

For each vertex $v_{n}$ we define the stencil $\mu(n) \subset\{1, \ldots, I\}$ consisting of the indices of the cells which share $v_{n}$ (first layer of cells). The stencil must gather at least, the indices of three different cells in order to apply the following method. In practice, if the first layer is not enough to satisfy the last consideration, we also include the neighbors of the cells which share $v_{n}$.

More specifically we have the following situations:

- if $v_{n}$ strictly belongs to $\Omega_{k}$, then the stencil only consists of cells of $\Omega_{k}$, $k=1,2$;

- if $v_{n}$ belongs to $\Gamma_{\mathrm{D}}$, then no stencil is required;

- if $v_{n}$ belongs to $\Gamma$ excluding the vertex associated to Dirichlet condition, we define $\mu_{1}(n)$ and $\mu_{2}(n)$ the stencils consisting of cells of $\Omega_{1}$ and $\Omega_{2}$, respectively;

- if $v_{n}$ belong to $\Gamma_{\mathrm{N}} \cap \Omega_{k}$, then $\mu(n)$ consists of cells belonging to $\Omega_{k}, k=1,2$. 


\subsection{The Interpolation Method}

Let us consider the vertex $v_{n}$ and its associated stencil $\mu(n)$. We then define $\psi_{n}$ as

$$
\psi_{n}=\sum_{i \in \mu(n)} \beta_{n i} \phi_{i},
$$

where we gather in vector $B^{n}=\left(\beta_{n i}\right)_{i \in \mu(n)}$ the coefficients of the linear combination of the cell data. As observed in [14] and [7], one has to reinforce the restriction such that equation (5) is consistent for first-degree polynomials. Let us define the operators

$$
f_{1}\left(B^{n}\right)=\sum_{i \in \mu(n)} \beta_{n i}, \quad f_{2}\left(B^{n}\right)=\sum_{i \in \mu(n)} \beta_{n i} x_{n i}, \quad f_{3}\left(B^{n}\right)=\sum_{i \in \mu(n)} \beta_{n i} y_{n i},
$$

where $\left(x_{n i}, y_{n i}\right)=\left(q_{i x}-v_{n x}, q_{i y}-v_{n y}\right)=v_{n} q_{i}$. We aim to choose vector $B^{n}$ such that

$$
f_{1}\left(B^{n}\right)=1, \quad f_{2}\left(B^{n}\right)=0, \quad f_{3}\left(B^{n}\right)=0 .
$$

Linear system (6) has a unique solution in very particular situations $(\# \mu(n)=$ 3 for instance) hence one has to design a strategy to determine a solution in the general cases. We propose here a new method based on the minimization of a functional. Let $\theta_{n}=\left(\theta_{n i}\right)_{i \in \mu(n)}$ be a set of values such that $\sum_{i \in \mu(n)} \theta_{n i}=1$ (the target values). We define the quadratic functional

$$
E\left(B^{n}\right)=\frac{1}{2} \sum_{i \in \mu(n)} \omega_{n i}\left(\beta_{n i}-\theta_{n i}\right)^{2}
$$

where $\omega_{n i}$ are strictly positive weights. We seek the unique vector $B^{n}$ which minimizes the quadratic functional (7) under constraints (6). Using the classical minimization method with the Lagrange multipliers, the problem turns to find vectors $\Lambda^{n}=\left(\lambda_{n 1}, \lambda_{n 2}, \lambda_{n 3}\right)$ and $B^{n}$ such that

$$
\nabla E\left(B^{n}\right)+\lambda_{n 1} \nabla f_{1}\left(B^{n}\right)+\lambda_{n 2} \nabla f_{2}\left(B^{n}\right)+\lambda_{n 3} \nabla f_{3}\left(B^{n}\right)=0 .
$$

We deduce that

$$
\beta_{n i}=\theta_{n i}-\frac{1}{\omega_{n i}}\left(\lambda_{n 1}+\lambda_{n 2} x_{n i}+\lambda_{n 3} y_{n i}\right), \quad i \in \mu(n) .
$$

Taking in consideration (6) and (8), we obtain the linear system

$$
\begin{gathered}
\lambda_{n 1} \sum_{i \in \mu(n)} \frac{1}{\omega_{n i}}+\lambda_{n 2} \sum_{i \in \mu(n)} \frac{x_{n i}}{\omega_{n i}}+\lambda_{n 3} \sum_{i \in \mu(n)} \frac{y_{n i}}{\omega_{n i}}=0, \\
\lambda_{n 1} \sum_{i \in \mu(n)} \frac{x_{n i}}{\omega_{n i}}+\lambda_{n 2} \sum_{i \in \mu(n)} \frac{x_{n i}^{2}}{\omega_{n i}}+\lambda_{n 3} \sum_{i \in \mu(n)} \frac{x_{n i} y_{n i}}{\omega_{n i}}=\sum_{i \in \mu(n)} \theta_{n i} x_{n i}, \\
\lambda_{n 1} \sum_{i \in \mu(n)} \frac{y_{n i}}{\omega_{n i}}+\lambda_{n 2} \sum_{i \in \mu(n)} \frac{x_{n i} y_{n i}}{\omega_{n i}}+\lambda_{n 3} \sum_{i \in \mu(n)} \frac{y_{n i}^{2}}{\omega_{n i}}=\sum_{i \in \mu(n)} \theta_{n i} y_{n i} .
\end{gathered}
$$


Assuming that $v_{n}$ and $q_{i}, i \in \mu(n)$, are non-collinear points, the linear system above has a unique solution $\Lambda^{n}$ from which we determine the coefficients of vector $B^{n}$ with equation (8). Several sets of target coefficients $\theta_{n i}$ and weights $\omega_{n i}$ will be proposed. For instance, a simple example may be

$$
\theta_{n i}=\frac{\left|c_{i}\right|}{\sum_{j \in \mu(n)}\left|c_{j}\right|}, \quad \omega_{n i}=1, \quad i \in \mu(n) .
$$

Notice that the Rauch, Batina, and Yang [14] corresponds to the case $\theta_{n i}=$ $\frac{1}{\# \mu(n)}$ and $\omega_{n i}=1$.

\subsection{Polynomial Reconstructions}

In order to compute an accurate approximation of $\mathcal{F}_{i j}$, we design the local polynomial approximations of the underlying solution involving vectors $\Phi$ and $\Psi$. As a first stage, let $S_{i j}$ be the stencil composed of the indices of the two vertices of the edge $e_{i j}, j \in\{1, \ldots, I, \mathrm{D}\}$. The second stage, detailed below, consists in defining the polynomial reconstructions based on the entries of vectors $\Phi$ and $\Psi$ associated to the appropriated stencils.

For a given inner edge $e_{i j}$, we design the first-degree polynomial

$$
\boldsymbol{\phi}_{i j}(x, y)=\phi_{i}+\mathcal{R}_{i j, x}\left(x-q_{i x}\right)+\mathcal{R}_{i j, y}\left(y-q_{i y}\right),
$$

where $\mathcal{R}_{i j, x}$ and $\mathcal{R}_{i j, y}$ are the coefficients to be determined. We denote by $\widetilde{\mathcal{R}}_{i j, x}$ and by $\widetilde{\mathcal{R}}_{i j, y}$ the unique coefficients such that the associated polynomial function $\widetilde{\boldsymbol{\phi}}_{i j} \equiv \widetilde{\boldsymbol{\phi}}_{i j}(x, y)$ interpolates $\phi_{i}$, defined on $q_{i}$, and $\psi_{n}$, defined on $v_{n}, n \in S_{i j}$. Notice that we also perform the polynomial $\widetilde{\boldsymbol{\phi}}_{j i} \equiv \widetilde{\boldsymbol{\phi}}_{j i}(x, y)$ using the reference cell point $q_{j}$ and the associated value $\phi_{j}$.

For an edge $e_{i \mathrm{D}} \subset \Gamma_{D}$, we proceed in the same way to provide polynomials $\widetilde{\boldsymbol{\phi}}_{i \mathrm{D}} \equiv \widetilde{\boldsymbol{\phi}}_{i \mathrm{D}}(x, y)$.

\section{Second-order Scheme}

In the previous section we introduce a technique to compute $\Psi$ from $\Phi$ and we design local polynomial reconstructions, based on vectors $\Psi$ and $\Phi$, in order to approximate the fluxes. We now return to the generic finite volume scheme (4) and we detail the computation of the numerical approximations $\mathcal{F}_{i j}$ and $f_{i}$.

\subsection{Diffusive Terms}

Having all the polynomial reconstructions in hand, we compute the numerical fluxes $\mathcal{F}_{i j}$ with respect to the interfaces of the cells of the mesh, as follows.

For an inner edge $e_{i j}$ in $\Omega_{1}$ or $\Omega_{2}$, we define the polynomials $\check{\boldsymbol{\phi}}_{i j}=\check{\boldsymbol{\phi}}_{j i}=$ $\sigma_{i j} \widetilde{\boldsymbol{\phi}}_{i j}+\sigma_{j i} \widetilde{\boldsymbol{\phi}}_{j i}$. We choose $\sigma_{i j}=\frac{\left|c_{i}\right|}{\left|c_{i}\right|+\left|c_{j}\right|}$ and $\sigma_{j i}=\frac{\left|c_{j}\right|}{\left|c_{i}\right|+\left|c_{j}\right|}$ for the sake of 
simplicity but situations with discontinuous diffusion coefficients for instance may require other expressions.

To compute the numerical approximation of the flux through an edge, we should take into account four situations:

- if $e_{i j}$ is a inner edge in $\Omega_{1}$ or $\Omega_{2}$, the numerical flux at the midpoint $m_{i j}$ writes

$$
\mathcal{F}_{i j}=-K\left(m_{i j}\right) \nabla \check{\boldsymbol{\phi}}_{i j}\left(m_{i j}\right) \cdot n_{i j} ;
$$

- for a Dirichlet boundary edge $e_{i \mathrm{D}}$, the numerical flux at the midpoint $m_{i \mathrm{D}}$ writes

$$
\mathcal{F}_{i \mathrm{D}}=-K\left(m_{i \mathrm{D}}\right) \nabla \widetilde{\boldsymbol{\phi}}_{i \mathrm{D}}\left(m_{i \mathrm{D}}\right) \cdot n_{i \mathrm{D}}
$$

- for a Neumann boundary edge $e_{i \mathrm{~N}}$, the numerical flux at the midpoint $m_{i \mathrm{~N}}$ writes

$$
\mathcal{F}_{i \mathrm{~N}}=g_{\mathrm{N}}\left(m_{i \mathrm{~N}}\right) ;
$$

- for an edge $e_{i j}$ on $\Gamma$, we denote by $k$ the index of one of the two domains and $k^{\prime}$ the other domain. We build the vertex value at $v_{n} \in \Gamma$ using $\mu(n)=\mu_{k}(n)$ and we compute the flux as

$$
\mathcal{F}_{i j}=-K_{k^{\prime}}\left(m_{i j}\right) \nabla \widetilde{\boldsymbol{\phi}}_{i j}\left(m_{i j}\right) \cdot n_{i j},
$$

where $c_{i}$ belongs to $\Omega_{k^{\prime}}$ and $c_{j}$ belongs to $\Omega_{k}$.

\section{$4.2 \quad$ Source Term}

Second-order approximations $f_{i}$ of the source term require an extra effort because these expressions do not derive from a flux. To derive second-order approximations, we split the cell $c_{i}$ into $\# \nu(i)$ triangular subcells denoted by $c_{i j}, j \in \nu(i)$, associated to edge $e_{i j}$.

The source term is evaluated using the approximation

$$
f_{i}=\frac{1}{\left|c_{i}\right|}\left[\sum_{j \in \nu(i)} \frac{\left|c_{i j}\right|}{3}\left(\sum_{n \in S_{i j}} f\left(v_{n}\right)+f\left(q_{i}\right)\right)\right],
$$

which can be simplified if $c_{i}$ is a triangular cell to $f_{i}=\frac{1}{3} \sum_{n \in S_{i}} f\left(v_{n}\right)$.

Remark 2. When $f$ is continuous piecewise on the cells, we compute the mean value of $f$ on $c_{i}$ using the continuity extension of $f$ at the vertex $v_{n} \in \nu(i)$. It results that we may have different values for the same vertex $v_{n}$ depending on the cell we are computing the mean value. 


\subsection{Residual Scheme}

Since $\mathcal{F}_{i j}$ and $f_{i}$ linearly depend on vector $\Phi$, we define the affine operator $\Phi \rightarrow \mathcal{G}_{i}(\Phi)$ for each cell $c_{i}, i=1, \ldots, I$, as

$$
\left.\mathcal{G}_{i}(\Phi)=\sum_{j \in \nu(i)} \frac{\left|e_{i j}\right|}{\left|c_{i}\right|} \mathcal{F}_{i j}(\Phi)\right)-f_{i},
$$

which corresponds to the finite volume scheme cast (4) in the residual form.

Gathering all the components $\mathcal{G}_{i}(\Phi)$ of the residual in vector $\mathcal{G}(\Phi)$, we obtain an affine operator from $\mathbb{R}^{I}$ into $\mathbb{R}^{I}$ such that vector $\Phi^{\star}$, solution of the problem $\mathcal{G}(\Phi)=0_{I}$, provides a constant piecewise approximation of the problem. We obtain a matrix-free scheme and the affine problem is solved by applying a GMRES procedure as explained in [6].

\section{$5 \quad$ Numerical Tests}

In this section we present several tests to quantitatively and qualitatively assess the robustness and accuracy of the proposed numerical scheme. In order to test the implementation of the method we check situations for which we manufacture a solution. All the simulations have been carried out on the academic domain $\Omega=] 0,1\left[^{2}\right.$.

Given the numerical approximation $\Phi^{\star}=\left(\phi_{i}^{\star}\right)_{i=1, \ldots, I}$ of a function $\phi$ on a mesh $\mathcal{T}$, we evaluate the error using the relative discrete $L^{2}$-norm given by

$$
E_{2}(\mathcal{T})=\left(\frac{\sum_{i=1}^{I}\left|c_{i}\right|\left(\phi\left(q_{i}\right)-\phi_{i}^{\star}\right)^{2}}{\sum_{i=1}^{I}\left|c_{i}\right| \phi\left(q_{i}\right)^{2}}\right)^{\frac{1}{2}}
$$

respectively. We also compute the convergence order of the error between two different meshes $\mathcal{T}_{1}$ and $\mathcal{T}_{2}$ as

$$
O_{2}\left(\mathcal{T}_{1}, \mathcal{T}_{2}\right)=2 \frac{\mid \log \left(E_{2}\left(\mathcal{T}_{1}\right) /\left(E_{2}\left(\mathcal{T}_{2}\right)\right) \mid\right.}{\left|\log \left(I_{1} / I_{2}\right)\right|}
$$

To analyse the maximum and minimum principle preservation we compute the maximum and the minimum of the numerical solution, including the solution on vertices $\Psi^{\star}=\left(\psi_{n}^{\star}\right)_{n=1, \ldots, N}$, as

$$
\operatorname{Max}=\max \left(\max _{i=1}^{I} \phi_{i}^{\star}, \max _{n=1}^{N} \psi_{n}^{\star}\right), \quad \operatorname{Min}=\min \left(\min _{i=1}^{I} \phi_{i}^{\star}, \min _{n=1}^{N} \psi_{n}^{\star}\right),
$$

respectively.

In all tests (except when explicitly mentioned), we assume that $q_{\ell}=m_{\ell}$, $\ell=1, \ldots, I$, the weights are $\omega_{n i}=1$, and the target coefficients $\theta_{n i}$ are given in (9). 


\subsection{Mild Anisotropy}

In the first test, we consider the homogeneous anisotropic tensor $K=\left[\begin{array}{ll}1.5 & 0.5 \\ 0.5 & 1.5\end{array}\right]$ and the solution

$$
\phi(x, y)=\frac{1}{2}\left[\frac{\sin ((1-x)(1-y))}{\sin (1)}+(1-x)^{3}(1-y)^{2}\right],
$$

on a square domain $\Omega=] 0,1\left[^{2}\right.$. The source term is manufactured from the exact solution and we prescribe the non-homogeneous Dirichlet condition $\phi_{\mathrm{D}}(x, y)=$ $\phi(x, y)$ on $\Gamma_{\mathrm{D}}=\partial \Omega$.
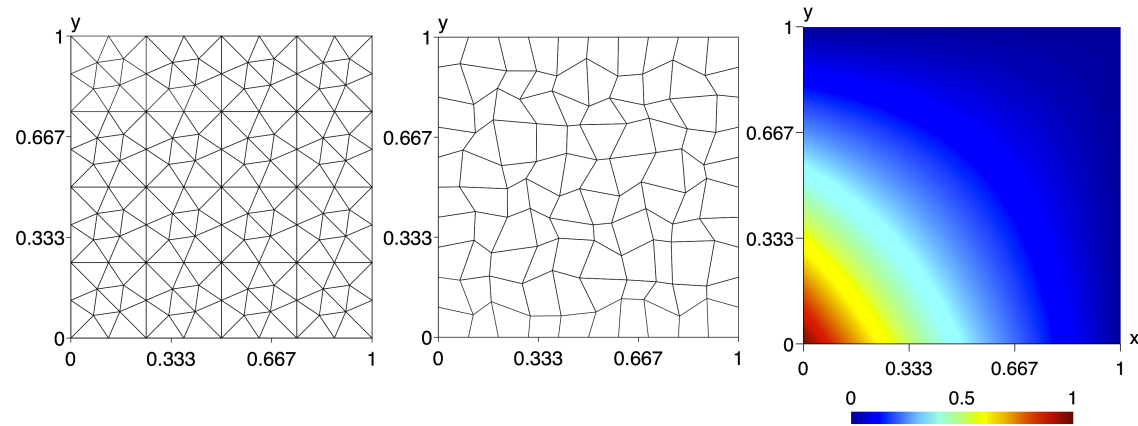

Fig. 2. Triangular mesh with acute angles (left), randomly deformed quadrilateral mesh (centre), and exact solution on a very fine mesh (right).

This test can be found in [22] and was originally proposed in [21] as a benchmark with a slightly modification in order to normalize the exact solution such that the minimum is equal to 0 .

Convergence Analysis. To perform the simulations and analyse the capacity of the scheme to provide accurate solutions with a second-order convergence, we use successive finer triangular meshes with strictly acute angles (see Fig. 2, left). In the same way, we use randomly deformed quadrilateral meshes (see Fig. 2, centre) to assess the robustness and the capacity of the scheme to handle complex meshes still providing second-order convergence rates.

These last meshes were obtained from structured meshes where we randomly move each inner vertex with a specific deformation factor (see the full procedure in [6]). We report in Table 1 the $L^{2}$-errors and convergence rates for the two classes of mesh and check an effective second-order of convergence for the method. 
Table 1. $L^{2}-$ norm error and convergence rates using triangular meshes with acute angles and deformed quadrangular meshes.

\begin{tabular}{ccccccc}
\hline \multirow{2}{*}{$I$} & \multicolumn{2}{c}{ Triangular meshes } & \multirow{2}{*}{$I$} & \multicolumn{2}{c}{ Deformed meshes } \\
\cline { 2 - 3 } \cline { 6 - 7 } & $E_{2}$ & $O_{2}$ & & $E_{2}$ & $O_{2}$ \\
\hline 224 & $4.97 \mathrm{E}-03$ & - & 400 & $8.16 \mathrm{E}-04$ & - \\
896 & $1.26 \mathrm{E}-03$ & 1.98 & & 1600 & $2.20 \mathrm{E}-04$ & 1.89 \\
3584 & $3.15 \mathrm{E}-04$ & 1.99 & 6400 & $5.43 \mathrm{E}-05$ & 2.02 \\
14336 & $7.91 \mathrm{E}-05$ & 2.00 & & 16900 & $2.11 \mathrm{E}-05$ & 1.95 \\
\hline
\end{tabular}

Maximum and Minimum Principle Preserving. The same problem was carried out with two distorted quadrangular meshes (see Fig.3, left and right) as proposed in [21] to check the maximum and minimum principle preservation.
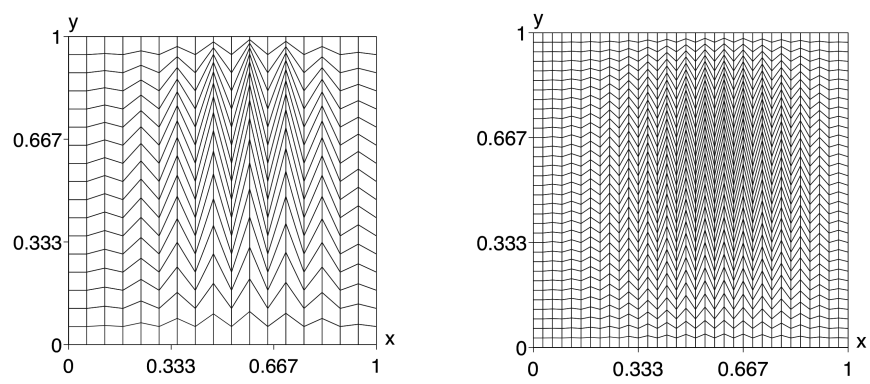

Fig. 3. Distorted quadrilateral meshes with $17 \times 17$ cells (left) and $33 \times 33$ cells.

Notice that the exact solution is located in the interval $[0,1]$. Table 2 shows the minimum and maximum of the solution and prove that the scheme preserves the expected bounded since the numerical solution ranges in the interval $[0,1]$.

Table 2. Minimum and maximum values of the numerical solution using distorted quadrangular meshes.

\begin{tabular}{cccc}
\hline$I$ & $N$ & Min & Max \\
\hline 289 & 324 & $0.00 \mathrm{E}+00$ & $1.00 \mathrm{E}+00$ \\
1089 & 1156 & $0.00 \mathrm{E}+00$ & $1.00 \mathrm{E}+00$ \\
\hline
\end{tabular}




\subsection{Heterogeneous Rotating Anisotropy}

In this test, we deal with a rotating and heterogeneous anisotropic tensor given by

$$
K(x, y)=\left[\begin{array}{cc}
\alpha x^{2}+y^{2} & (\alpha-1) x y \\
(\alpha-1) x y & x^{2}+\alpha y^{2}
\end{array}\right], \quad \alpha \geqslant 1 .
$$

The exact solution is $\phi(x, y)=\exp \left(-20 \pi\left((x-0.5)^{2}+(y-0.5)^{2}\right)\right)$ (see Fig. 4, centre) and the source term $f$ is computed from the exact solution applying the operator. We prescribe a full Dirichlet boundary condition $\left(\Gamma_{\mathrm{D}}=\partial \Omega\right)$ with nonhomogeneous Dirichlet condition $\phi_{\mathrm{D}}(x, y)=\phi(x, y)$ on $\Gamma_{\mathrm{D}}$. As described in [22], the source term $f$ corresponds to an injection at the center of $\Omega$, between two sinks (see Fig. 4, right). Clearly, the magnitude of the injection as well as the sinks increase as $\alpha$ increase. Moreover, the two eigenvalues of $K$ are $\lambda_{1}(x, y)=x^{2}+y^{2}$ and $\lambda_{2}(x, y)=\alpha\left(x^{2}+y^{2}\right)$, which correspond to an anisotropic ratio equal to $\alpha$ in the whole domain, and we have $\inf _{(x, y) \in \Omega} \lambda_{2}=0$ and $\sup _{(x, y) \in \Omega} \lambda_{2}=2 \alpha$. Given that, for large values of $\alpha$ we have a highly heterogeneous case which can induces numerical locking. As in [22] we use successive finer structured triangular
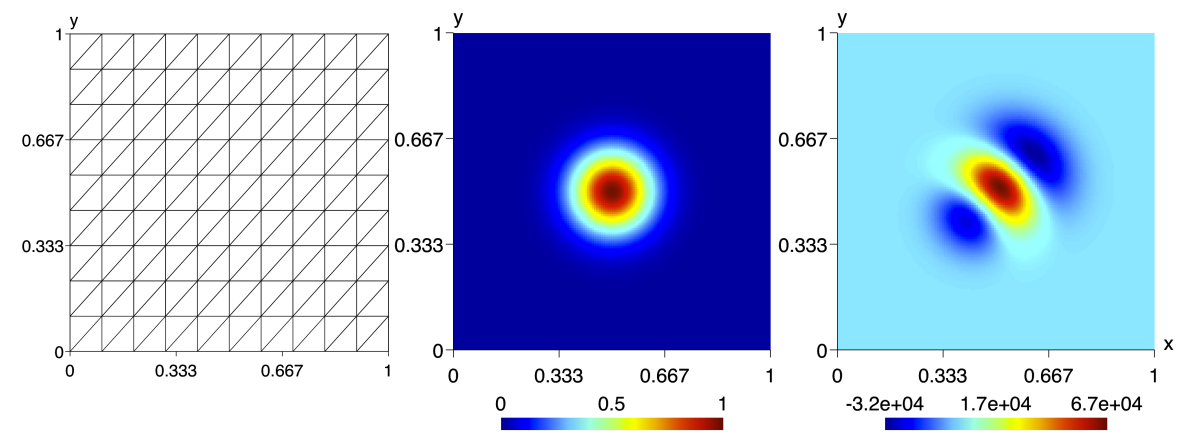

Fig. 4. An example of a structured triangular mesh (left), the exact solution on a very fine mesh (centre), and the source term for $\alpha=1000$ (right).

meshes (see Fig. 4, left) to evaluate the convergence rates and the maximum and minimum principle preserving for $\alpha \in\{10,100,1000\}$. We present in Table 3 the $L^{2}$-error and the convergence rates for three values of $\alpha$. We get an effective second-order of convergence and no numerical locking is reported.

\subsection{Numerical Locking Test}

We consider the anisotropic tensor $K=\left[\begin{array}{ll}1 & 0 \\ 0 & \delta\end{array}\right]$, where $\delta>0$, the exact solution is taken to be $\phi(x, y)=\sin (2 \pi x) \exp \left(\frac{-2 \pi}{\sqrt{\delta}} y\right)$ and the source term is $f(x, y)=0$ 
Table 3. $L_{2}$-norm errors and convergence rates for three values of $\alpha$ using structured triangular meshes.

\begin{tabular}{ccccccccc}
\hline \multirow{2}{*}{$I$} & \multicolumn{2}{c}{$\alpha=10$} & & \multicolumn{2}{c}{$\alpha=100$} & & \multicolumn{2}{c}{$\alpha=1000$} \\
\cline { 2 - 3 } \cline { 8 - 9 } & $E_{2}$ & $O_{2}$ & & $E_{2}$ & $O_{2}$ & & $E_{2}$ & $O_{2}$ \\
\hline 180 & $1.12 \mathrm{E}-01$ & - & & $1.39 \mathrm{E}-01$ & - & & $1.44 \mathrm{E}-01$ & - \\
760 & $2.26 \mathrm{E}-02$ & 2.22 & & $2.89 \mathrm{E}-02$ & 2.18 & & $3.04 \mathrm{E}-02$ & 2.16 \\
3120 & $5.40 \mathrm{E}-03$ & 2.03 & & $7.02 \mathrm{E}-03$ & 2.00 & & $7.62 \mathrm{E}-03$ & 1.96 \\
12640 & $1.33 \mathrm{E}-03$ & 2.01 & & $1.74 \mathrm{E}-03$ & 1.99 & & $1.95 \mathrm{E}-03$ & 1.95 \\
\hline
\end{tabular}

on a square domain $\Omega=] 0,1\left[^{2}\right.$. Note that $\phi$ depends on $\delta$ such that the solution is almost constant in the $y$-direction for large values of $\delta$. We consider three situations:

- Case A: we prescribe a Dirichlet boundary data with non-homogeneous Dirichlet condition on $\Gamma_{\mathrm{D}}=\partial \Omega$ and $\Gamma_{\mathrm{N}}=\varnothing$;

- Case B: we prescribe a mixed Dirichlet-Neumann boundary data with the conditions $\phi_{\mathrm{D}}(x, y)=\phi(x, y)$ on $\Gamma_{\mathrm{D}}=\{(x, y): x=0 \vee y=0\}$ and $g_{\mathrm{N}}=$ $-K \nabla \phi \cdot n$ on $\Gamma_{\mathrm{N}}=\{(x, y): x=1 \vee y=1\}$;

- Case C: we prescribe a pure Neumann boundary data $g_{\mathrm{N}}=-K \nabla \phi \cdot n$ on $\Gamma_{\mathrm{N}}=\partial \Omega$ and $\Gamma_{\mathrm{D}}=\varnothing$. Since we only use the Neumann condition, the operator is singular and an extra condition will be provided to ensure the uniqueness, For example, one can impose the solution to have zero mean value on the whole domain. In practice, such property is achieved by using the GMRES solver without introducing explicitly the zero mean value condition.

We carried out the simulations for a mild anisotropic diffusion case with $\delta=10$, for a high anisotropic diffusion case with $\delta=10^{3}$, and at last we set $\delta=10^{6}$ for a very high anisotropic diffusion case. Notice that for large values of $\delta$ the diffusion occurs mainly in the $y$-direction while the information is transmitted mainly in the $x$-direction (see Fig. 5). Due this fact, some numerical schemes have difficulties to achieve an accurate solution and the expected convergence order. We cite $[22,23]$ and the benchmark [21]. We report in Tables 4, 5, and 6 the results for cases $\mathrm{A}, \mathrm{B}$, and $\mathrm{C}$ with the three values of $\delta$.

Table 4. $L^{2}$-norm errors and convergence rates for case A with three values of $\delta$.

\begin{tabular}{|c|c|c|c|c|c|c|}
\hline \multirow{2}{*}{$I$} & \multicolumn{2}{|c|}{$\delta=10$} & \multicolumn{2}{|c|}{$\delta=10^{3}$} & \multicolumn{2}{|c|}{$\delta=10^{6}$} \\
\hline & $E_{2}$ & $\mathrm{O}_{2}$ & $E_{2}$ & $\mathrm{O}_{2}$ & $E_{2}$ & $\mathrm{O}_{2}$ \\
\hline 896 & $1.44 \mathrm{E}-02$ & - & $1.12 \mathrm{E}-02$ & - & $1.11 \mathrm{E}-02$ & - \\
\hline 3584 & $3.51 \mathrm{E}-03$ & 2.04 & $2.46 \mathrm{E}-03$ & 2.19 & $2.41 \mathrm{E}-03$ & 2.21 \\
\hline 14336 & $8.64 \mathrm{E}-04$ & 2.02 & $5.79 \mathrm{E}-04$ & 2.09 & $5.64 \mathrm{E}-04$ & 2.10 \\
\hline 57344 & $2.14 \mathrm{E}-04$ & 2.01 & $1.42 \mathrm{E}-04$ & 2.03 & $1.38 \mathrm{E}-04$ & 2.04 \\
\hline
\end{tabular}



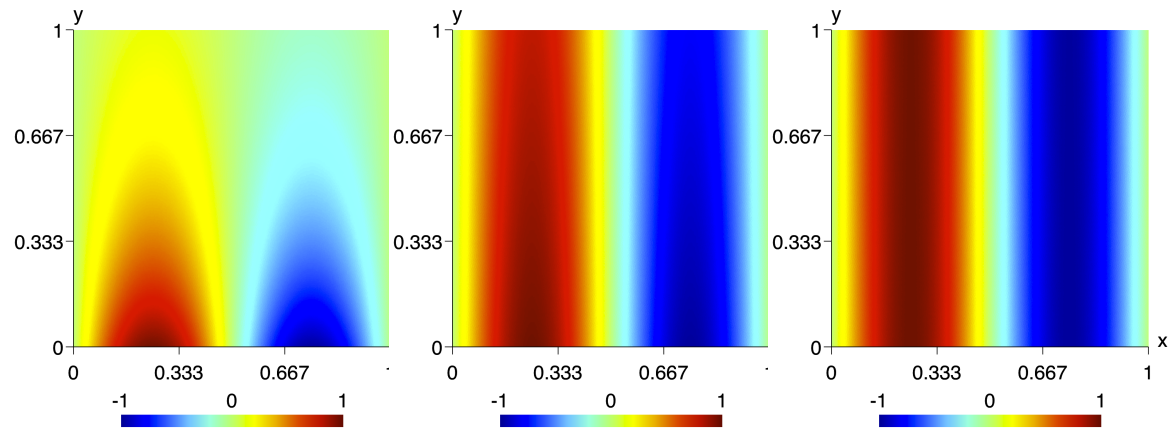

Fig. 5. Exact solution on a very fine mesh mesh with $\delta=10$ (left), $\delta=10^{3}$ (centre), and $\delta=10^{6}$ (right).

Table 5. $L^{2}$-norm errors and convergence rates for case B with three values of $\delta$.

\begin{tabular}{|c|c|c|c|c|c|c|}
\hline \multirow{2}{*}{$I$} & \multicolumn{2}{|c|}{$\delta=10$} & \multicolumn{2}{|c|}{$\delta=10^{3}$} & \multicolumn{2}{|c|}{$\delta=10^{6}$} \\
\hline & $E_{2}$ & $\mathrm{O}_{2}$ & $E_{2}$ & $\mathrm{O}_{2}$ & $E_{2}$ & $\mathrm{O}_{2}$ \\
\hline 896 & $7.07 \mathrm{E}-03$ & - & $1.70 \mathrm{E}-02$ & - & $1.91 \mathrm{E}-02$ & - \\
\hline 3584 & $1.64 \mathrm{E}-03$ & 2.11 & $2.55 \mathrm{E}-03$ & 2.74 & $2.79 \mathrm{E}-03$ & 2.77 \\
\hline 14336 & $4.05 \mathrm{E}-04$ & 2.02 & $5.35 \mathrm{E}-04$ & 2.25 & $7.12 \mathrm{E}-04$ & 1.97 \\
\hline 57344 & $1.07 \mathrm{E}-04$ & 1.92 & $1.26 \mathrm{E}-04$ & 2.09 & $1.67 \mathrm{E}-04$ & 2.09 \\
\hline
\end{tabular}

Table 6. $L^{2}$-norm errors and convergence rates for case $\mathrm{C}$ with three values of $\delta$.

\begin{tabular}{|c|c|c|c|c|c|c|}
\hline \multirow{2}{*}{$I$} & \multicolumn{2}{|c|}{$\delta=10$} & \multicolumn{2}{|c|}{$\delta=10^{3}$} & \multicolumn{2}{|c|}{$\delta=10^{6}$} \\
\hline & $E_{2}$ & $\mathrm{O}_{2}$ & $E_{2}$ & $\overline{\mathrm{O}_{2}}$ & $E_{2}$ & $\overline{\mathrm{O}_{2}}$ \\
\hline 224 & $7.20 \mathrm{E}-02$ & - & $3.75 \mathrm{E}-01$ & - & $4.20 \mathrm{E}-01$ & - \\
\hline 896 & $1.47 \mathrm{E}-02$ & 2.29 & $6.46 \mathrm{E}-02$ & 2.54 & $7.21 \mathrm{E}-02$ & 2.54 \\
\hline 3584 & $3.49 \mathrm{E}-03$ & 2.08 & $1.39 \mathrm{E}-02$ & 2.22 & $2.16 \mathrm{E}-02$ & 1.7 \\
\hline 14336 & $8.62 \mathrm{E}-04$ & 2.02 & $3.61 \mathrm{E}-03$ & 1.94 & - & 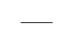 \\
\hline
\end{tabular}

When including Dirichlet condition on a part of the domain, no numerical locking phenomenon is observed and we get the optimal convergence order. For the pure Neumann case, no numerical locking appears for $\delta=10$ and $\delta=10^{3}$ and we also get a second-order convergence rate. For $\delta=10^{6}$ (the worst case), we face to difficulties to get the full convergence but, on the contrary of the most of finite volume schemes, we overcome the numerical locking and get a rather good convergence rate. The two last results for this test are not shown due the inability of the GMRES procedure to return an acceptable residual. We highlight that for such a simulation, the preconditioning matrix choice is of crucial importance. 


\subsection{Discontinuous Anisotropy with the Continuity Condition}

We now turn to the situation where the diffusion tensor is discontinuous across the interface $\Gamma$. We consider the domain $\Omega=\Omega_{1} \cup \Omega_{2}$ with $\left.\Omega_{1}=\right] 0,0.5[\times] 0,1[$ and $\left.\Omega_{2}=\right] 0.5,1[\times] 0,1[$ while the interface is given by $\Gamma=\{0.5\} \times] 0,1[$ (see Fig. 6). The exact solution is given by $\phi_{1}(x, y)=\cos (\pi x) \sin (\pi y)$ and $\phi_{2}(x, y)=$ $0.01 \cos (\pi x) \sin (\pi y)$, and the diffusion tensor is given by

$$
K_{1}=\left[\begin{array}{ll}
1 & 0 \\
0 & 1
\end{array}\right], \quad K_{2}=\left[\begin{array}{cc}
100 & 0 \\
0 & 0.01
\end{array}\right],
$$

on $\Omega_{1}$ and $\Omega_{2}$, respectively. The source terms are $f_{1}(x, y)=2 \pi^{2} \cos (\pi x) \sin (\pi y)$ and $f_{2}(x, y)=\left(1+0.01^{2}\right) \pi^{2} \cos (\pi x) \sin (\pi y)$ and we set $\Gamma_{\mathrm{D}}=\partial \Omega$ with Dirichlet condition $\phi_{\mathrm{D}}(0, y)=\sin (\pi y)$ on the left side, $\phi_{\mathrm{D}}(1, y)=0.01 \sin (\pi y)$ on the right side, and $\phi_{\mathrm{D}}(x, 0)=\phi_{\mathrm{D}}(x, 1)=0$ on the other sides. At last, we prescribe the continuity condition $\phi_{1}=\phi_{2}$ on interface $\Gamma$. The test was initially proposed in [24].
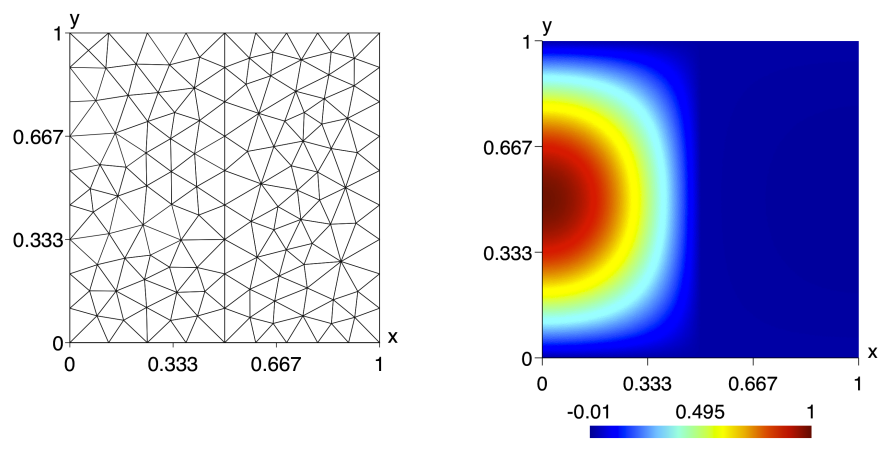

Fig. 6. Regular triangular Delaunay mesh in both subdomains $\Omega_{1}$ and $\Omega_{2}$ (left), and exact solution on a very fine mesh (right).

Table 7. $L_{2}$-norm errors and convergence rates when the vertices on $\Gamma$ are computed with the cells from $\Omega_{1}$ using $\mu(n)=\mu_{1}(n)$ and from $\Omega_{2}$ using $\mu(n)=\mu_{2}(n)$

\begin{tabular}{cccccc}
\hline \multirow{2}{*}{$I$} & \multicolumn{2}{c}{$\mu(n)=\mu_{1}(n)$} & & \multicolumn{2}{c}{$\mu(n)=\mu_{2}(n)$} \\
\cline { 2 - 3 } \cline { 5 - 6 } & $E_{2}$ & $O_{2}$ & & $E_{2}$ & $O_{2}$ \\
\hline 236 & $7.38 \mathrm{E}-03$ & - & $6.82 \mathrm{E}-03$ & - \\
936 & $1.80 \mathrm{E}-03$ & 2.05 & & $1.58 \mathrm{E}-03$ & 2.13 \\
3962 & $4.13 \mathrm{E}-04$ & 2.04 & & $3.96 \mathrm{E}-04$ & 1.92 \\
17010 & $9.13 \mathrm{E}-05$ & 2.07 & & $8.83 \mathrm{E}-05$ & 2.06 \\
\hline
\end{tabular}


We have performed two type of tests. In a first run, the reconstructions for the vertices $v_{n}$ on $\Gamma$ are carried out with the cells from $\Omega_{1}$ using $\mu(n)=$ $\mu_{1}(n)$ while the gradient is computed with the function $\widetilde{\phi}_{i j}$ located on the cell $c_{i}$ belonging to the domain $\Omega_{2}$. The second run provides a numerical solution where we take $\mu(n)=\mu_{2}(n)$ and the gradient is computed with the data of cell $c_{i} \subset \Omega_{1}$. We present in Table 7 the two situations and we observe that the scheme provides the same accuracy. Nevertheless, our numerical experience highlights that the number of GMRES iteration is larger (two times) when dealing with the first case, i.e. the problem needs a higher computer effort when the gradient is computed on the side where the diffusion tensor is larger.

\section{Conclusion}

An extension of the original Frink's method has been proposed and implemented to perform the cell-to-vertex reconstruction applied in the context of anisotropic and discontinuous diffusion tensors. The method enables to associate the cell value at any location point inside the cell still preserving both the second-order accuracy and the robustness, even for difficult diffusion problem such as numerical locking. Several numerical experiences have been carried out to assess the method performance and demonstrate the capacity to handle a wide range of situations in the context of the anisotropic and discontinuous diffusion equation.

\section{Acknowledgements}

The paper was supported by the Research Centre of Mathematics of the University of Minho with the Portuguese Funds from the Fundação para a Ciência e a Tecnologia, through the Project PEstOE/MAT/ UI0013/2014 and by FEDER Funds through Programa Operacional Factores de Competitividade - COMPETE and by Portuguese Funds through FCT - Fundação para a Ciência e a Tecnologia, within the Project PTDC/MAT/121185/2010.

\section{References}

1. Berthon, C., Coudière, Y., Desveaux, V.: Second-order MUSCL schemes based on dual mesh gradient reconstrution (DMGR), to be published in ESAIM: Mathematical Modelling and Numerical Analysis (2014).

2. Bertolazzi, E., Manzini, G.: Least square-based finite volumes for solving the advectiondiffusion of contaminants in porous media, Applied Numerical Mathematics 51, 451-461 (2004).

3. Bertolazzi, E., Manzini, G.: On vertex reconstructions for cell-centered finite volume approximations of $2 \mathrm{D}$ anisotropic diffusion problems, Math. Models and Meth. in App. Sci. 17 (1), 1-32 (2007).

4. Costa, R., Clain, S., Machado, G. J.: New cell-vertex reconstruction for finite volume scheme: application to the convection-diffusion-reaction equation, under review. 
5. Chandrashekar, P., Garg, A.: Vertex-centroid finite volume scheme on tetrahedral grids for conservation laws, Computers and Mathematics with Applications 65, 5874 (2013).

6. Clain, S., Machado, G. J., Nóbrega, J. M., Pereira, R. M. S.: A sixth-order finite volume method for the convection-diffusion problem with discontinuous coefficients, Computer Methods in Applied Mechanics and Engineering 267, 43-64 (2013).

7. Coudière, Y., Vila, J.P., Villedieu, P.: Convergence rate of a finite volume scheme for a two dimensional convection-diffusion problem, M2AN 3 (33), 493-516 (1999).

8. Domelevo, K., Omnes, P.: A finite volume method for the Laplace equation on almost arbitrary two-dimensional grids, M2AN Math. Model. Numer. Anal. 39, 1203-1249 (2005).

9. Hermeline, F.: A finite volume method for the approximation of diffusion operators on distorted meshes, J. Comput. Phys. 160, 481-499 (2000).

10. Hidalgo, A., Dumbser, M.: ADER Schemes for Nonlinear Systems of Stiff Advection-Diffusion-Reaction Equations, J. Sci. Comput. 48 (1-3), 173-189 (2011).

11. Holmes, D.G., Connel, S.D.: Solution of the 2D Navier-Stokes equations on unstructured adaptive grids, AIAA Paper 89-1392, 1989.

12. Ivan, L., Groth, C.P.T.: High-order solution-adaptative central essentially nonoscillatory (CENO) method for viscous flows, AIAA Paper 2011-367, 2011.

13. Ollivier-Gooch, C.: High-Order ENO schemes for unstructured Meshes based on least-squares reconstruction, AIAA Paper 97-0540, 1997.

14. Rausch, R.D., Batina, J.T., Yang, H.T.Y.: spatial adaptation procedure on unstructured meshes for accurate unsteady aerodynamic flow computation, AIAA Paper 91-1106, 1991.

15. Frink, N.T.: Three-dimensional upwind scheme for solving the Euler equations on unstructured tetrahedral grids, Ph. D Dissertation, Virginia Polytechnic Institute and state university (1991).

16. Frink, N.T.: Upwind scheme for solving the Euler equations on unstructured tetrhedral meshes, AIAA Journal 1, 70-77 (1992).

17. Frink, N.T.: Recent progress toward a three-dimensional unstructured NavierStokes flow solver, AIAA Paper 94-0061, 1994.

18. Jawahar, P., Kamath, H.: A high-resolution procedure for Euler and Navier-Stokes computations on unstructured Grids, J. Comp. Phys. 164 (2000) 165-203.

19. Eymard, R., Gallouët, T., Herbin, R.: Finite volume approximation of elliptic problems and convergence of an approximate gradient, Applied Numerical Mathematics 37, 31-53 (2001).

20. Ollivier-Gooch, C., Van Altena, M.: A high-order-accurate unstructured mesh finite-volume scheme for the advection-diffusion equation, Journal of Computational Physics Archive 181 (2), 729-752 (2002).

21. Herbin, R., Hubert, F.: Benchmark on discretization schemes for anisotropic diffusion problems on general grids. In Finite Volumes for Complex Applications V, 659-692 (2008).

22. Gao, Z., Wu, J.: A linearity-preserving cell-centred scheme for the heterogeneous and anisotropic diffusion equations on general meshes. Int. J. Numer. Meth. Fluids 67, 21572183 (2011).

23. Manzini, G., Putti, M.: Mesh locking effects in the finite volume solution of 2D anisotropic diffusion equations. Journal of Computational Physics 220, 751-771 (2007).

24. Christophe, L.P., Hai, O.T.: A Cell-Centered Scheme For Heterogeneous Anisotropic Diffusion Problems On General Meshes. IJFV International Journal On Finite Volumes 8, 1-40 (2012). 